\title{
Osseointegration- A Review
}

\author{
Prutha Vaidya ${ }^{1}$,Swapna Mahale ${ }^{2}$,Sunila Kale ${ }^{3}$,Agraja Patil ${ }^{4}$ \\ ${ }_{1,3,4}$ (Post Graduate Student, Department of Periodontics, MGV KBH Dental College, Nashik) \\ ${ }_{2}^{2}$ (Professor and HOD, Department of Periodontics, MGV KBH Dental College, Nashik)
}

\begin{abstract}
The successful replacement of lost natural teeth by tooth root analogues is a major advancement in the field of dentistry. The success greatly depends on adequate integration of these implants within the bone. This bone-implant integration is known as osseointegration. The science of osseointegration has widened the scope of treatment options for edentulous patients.
\end{abstract}

Keywords : Osseointegration, Osseodensification, Implants, Bone

\section{Introduction}

Osseointegration comes from the Greek word 'osteon' meaning bone, and the Latin word 'integrare', meaning to make whole. Branemark defined it "as a direct contact between the bone and metallic implants, without interposed soft tissues layers" (1969). Later it was modified (1977) "as a direct structural and functional connection between ordered, living bone and the surface of a load carrying implant".

American Academy of Implant Dentistry (1986) defined it as contact established without interposition of nonbone tissue between normal remodeled bone and an implant entailing a sustained transfer and distribution of load from implant to and within the bone tissue.

Meffert et al. (1987) subdivided into:

- Adaptive Osseointegration: Osseous tissue approximating the surface of the implant without apparent soft tissue interface at light microscopic level.

- Biointegration: Is a direct biochemical bone surface attachment confirmed at electron microscopic level.

Zarb and T. Albrektsson (1991) defined it as a process whereby clinically asymptomatic rigid fixation of alloplastic materials is achieved and maintained, in bone during functional loading.

\section{History Of Osseointegration}

Dr. Per Ingvar Branemark, an anatomist is credited as the person who has coined the term "osseointegration". Branemark along with his team was working in the laboratory of the vital microscopy (1952), laboratory of experimental Biology, University of Goteberg Sweden, (1960), Institute of Applied biotechnology, Goteberg (1978). ${ }^{[1]}$ The main study of his group was to understand the mechanism of bone healing and bone response to the thermal, mechanical, chemical injuries by using vital microscopy.

In their study the titanium (Ti) chambers were used for placing the vital microscope into the rabbit's fibula. After the studying of the bone biomechanics in one animal, the team used to recover the vital microscope and place it into the other animal model. While recovering Branemark observed that the Ti chambers were firmly adherent to the bone. By this observation they concluded that the titanium was firmly integrated to the bone.

By observing this property the integration between the bone and $\mathrm{Ti}$ screws was termed as "osseointegration". In 1965, first human edentulous patient was treated by using the Ti screws (implants) by reconstruction of resorbed edentulous arches using autologous tibial bone grafts.

\section{Mechanism Of Osseointegration}

After the surgical placement of implants into endosteal location, the traumatized bone around these implants begins the process of wound healing.

It can be separated into

-Inflammatory phase

-Proliferative phase

-Maturation phase

\subsection{Inflammatory phase}

Vascular events: When platelets come in contact with synthetic surfaces, they release serotonin and histamine causing further platelet aggregation and thrombosis. The clotting cascade is initiated as soon as blood contacts proteins or a foreign material causing blood coagulation. 
Cellular events: It is non-specific in the beginning consisting majorly of neutrophils that peak during 3 to 4 days of surgery but towards the end of the first week, the generalized inflammatory response becomes more specific in nature consisting majorly of increasing numbers of thymus dependent lymphocytes ( $\mathrm{T}$ cells), B cells, killer $(\mathrm{K})$ cells, natural killer (NK) cells and macrophages.

\subsection{Proliferative Phase}

During this phase, vascular ingrowth occurs from the surrounding vital tissues, a process called neovascularization. Metabolism of the local inflammatory cells, fibroblasts, progenitor cells and other local cells creates an area of relative hypoxia in the wound area which triggers the local mesenchymal cells to differentiate into fibroblasts, osteoblasts and chondroblasts. An extracellular matrix is laid down by these cells and eventually a fibro-cartilaginous callus is formed that transforms to bone callus. The initial immature bone is called the woven bone.

\subsection{Maturation Phase}

Appositional woven bone is laid down on the scaffold of necrotic bone in the peri-implant space that resulted from operative trauma by differentiated mesenchymal cells in the advancing granulation tissue mass. This process occurs concurrently with the ossification of the fibrocartilaginous callus noted previously. Simultaneous resorption of these "composite" trabeculae and the newly formed bone, coupled with the deposition of mature concentric lamellae eventually results in complete bone remodeling, leaving a zone of living lamellar bone that is continuous with the surrounding basal bone.

\section{Osteogenesis}

The phenomenon by which bone can form over an implant surface was described by Osborn and Newsley in $1980 .^{[2]}$ They described that this could occur via two means:

- Contact osteogenesis: In contact osteogenesis, new bone forms first on the implant surface. The implant surface has to be colonized by bone cells before the beginning of bone matrix formation.

- Distance osteogenesis: In distance osteogenesis, new bone is formed on the surfaces of old bone in the peri-implant site.

\subsection{Implant and bone interface}

\section{Implant Tissue Interface}

Scanning electron microscopic study of the interface shows that parallel alignment of the lamellae of haversian system of the bone next to the Ti implants. No connective tissue or dead space was observed at the interface.

\subsubsection{Mechanism of attachment}

The cells bind to each other or any other foreign materials by a layer of extracellular macro molecules (glycoproteins). At the molecular level the macromolecules contains Tri-peptides made up of Arginin-glycinAspartic acid (RGD). The cells like fibroblasts and other connective tissue cells contain binding elements called as "integrins". The integrins recognizes the RGDs and bind to them.

\subsection{Implant connective tissue interface}

The supra-crestal connective tissue fibers are arranged parallel to the surface of the implant making the attachment not as strong as that of the connective tissue and tooth interface. But the implant connective tissue interface is strong enough to withstand the occlusal forces and microbial invasions.

\subsection{Implant epithelial interface}

The implant epithelial interface is considered as Biologic seal by many authors. At this interface the glycoprotein layer is adherent to the implant surface to which hemidesmosomes are attached which is almost similar to the junctional epithelium.

\section{Factors Influencing Osseointegration}

Factors that affect the bone anchorage of an implanted device are implant biocompatibility, design characteristics, surface characteristics, the state of the host bed, the surgical technique and the loading conditions. $^{[3]}$

There have been various advancements on the above mentioned fronts to ensure reliable bone anchorage of an implant. New materials, designs or surfaces have been introduced with simultaneous claims of these being superior to those used in the past. Despite of some relevance for biomaterials research, this approach has not been without drawbacks. Improvements of the surgical technique seem to be a reliable way of increasing 
oral implant success. A new surgical technique has been recently developed for placement of implants that requires no removal of bone to place the implant and leads to enhanced stability and increased bone density. This technique is known as osseodensification.

Osseodensification is a nonextraction technique which was developed by Huwais in $2013 .{ }^{[4]}$ It is carried out using specially designed burs to increase bone density as they expand an osteotomy.

Standard drill designs excavate bone to create space for implant placement. Unlike traditional bone drilling technologies, osseodensification does not excavate bone tissue. On the contarary, it preserves bone bulk, so bone tissue is simultaneously compacted and autografted in an outwardly expanding direction to form the osteotomy. It is accomplished by using proprietary densifying burs. When the densifying bur is rotated at high speed in a reversed, non-cutting direction with steady external irrigation (Densifying Mode), a dense compacted layer of bone tissue is formed along the walls and base of the osteotomy. ${ }^{[5]}$ The goal in implant placement is to achieve primary implant stability. Implant primary mechanical stability is directly related to surrounding bone quality and quantity. Maintaining and preserving bone during osteotomy preparation leads to increased primary mechanical stability, increased bone to implant contact (BIC), which then enhances implant secondary stability, and accelerates healing.

Trisi et al. ${ }^{[6]}$ conducted an animal study on sheep model to evaluate this technique and its effect on bone density, ridge width and implant secondary stability. They observed no implant failures after 2 months of healing. There was a significant increase of ridge width and bone volume percentage (\%BV) (approximately $30 \%$ higher) and also a significantly better removal torque values and micromotion under lateral forces.

\subsection{Invasive methods}

\section{Methods Of Evaluation Of Osseointegration}

1. Histological sections

2. Histomorphometric

3. Transmission electron microscopy

4. Pull out tests.

5. By using torque gauges

Historically, microscopic or histologic analysis has been considered as the gold standard method to evaluate the degree of osseointegration. However, due to the invasiveness of this method and related ethical issues, various other methods of analysis have been proposed.

\subsection{Non-invasive methods}

1. Percussion test: An osseointegrated implant makes a ringing sound on percussion whereas an implant that has undergone fibrous integration produces a dull sound.

2. Radiographs

3. Reverse torque test: A reverse or unscrewing torque is applied to assess implant stability at the time of abutment connection. Implants that rotate under the applied torque are considered failures and are then removed.

4. Periotest: It is a device which is an electrically driven and electronically monitored tapping head that percusses the implant a total of 16 times in about $4 \mathrm{~s}$.

5. Resonance frequency analysis: It measures implant stability and bone density at various time points using vibration and structural principle analysis. Classically, the implant stability quotient (ISQ) has been found to vary between 40 and 80 , the higher the ISQ, the higher the implant stability. It is inversely proportional to the resonance frequency. Implant stability can be determined for implants with an ISQ of 47 . All implants with an ISQ more than 49 osseointegrated when left to heal for 3 months. All implants with an ISQ more than 54 osseointegrated when immediately loaded.

\subsection{Evaluation of success of osseointegration}

\section{Alberktsson Success Criteria (1986) ${ }^{[7]}$}

1. The individual unattached implant should be immobile when tested clinically.

2. The radiographic evaluation should not show any evidence of radiolucency.

3. The vertical bone loss around the fixtures should be less than $0.2 \mathrm{~mm}$ per year after first year of implant loading.

4. The implant should not show any signs of pain, infection, neuropathies, parasthesia, violation of mandible canals and sinus drainage.

5. The success rate of $85 \%$ at the end of 5 year and $80 \%$ at the end of 10 years. 
According to the present concepts the width of the attached gingival, co- existing medical conditions, smoking, width of the implant, suture material used, all play an important role in implant success. Even genetic and immunological factors like TNF- $\alpha$ and IL- $1 \beta$ have been identified as markers for implant success. ${ }^{[8]}$

\section{Conclusion}

Osseointegration is one of the most critical aspects in implant success. Successful osseointegration is a mandatory for functional dental implants. It is necessary to be aware of the mechanism of osseointegration in order to direct research to enhance success. Various researchers have focused on the hardware aspect of an implant to yield better results, however the recent developments in surgical techniques such as osseodensification have opened new arrays for research into the field of implants.

\section{References}

[1]. Branemark R, Branemark PI, Rydevik B, Myers RR. Osseointegration in skeletal reconstruction and rehabilitation: A review. JRRD 2001;38(2):175-81.

[2]. Osborn JF, Newesely H. Dynamic aspects of the implant bone interface. In: Heimke G, ed. Dental implants: materials and systems. München. Carl Hanser Verlag 1980:111-23.

[3]. Albrektsson T, Branemark P-I, Hansson H-A, Lindstrom J. Osseointegrated titanium implants. Requirements for ensuring a longlasting direct bone-to-implant anchorage in man. Acta Orthop Scand 1981;52:155-170.

[4]. Huwais S, Meyer E. Osseodensification: A novel approach in implant o preparation to increase primary stability, bone mineral density and bone to implant contact. Int J Oral Maxillofac Implants. 2015.

[5]. EG, Huwais S. Osseodensification Is A Novel Implant Preparation Technique That Increases Implant Primary Stability By Compaction and Auto-Grafting Bone. American Academy of Periodontology. [abstract]. San Francisco, CA. 2014.

[6]. Trisi P, Berardini M, DDS, Falco A, PhD, DDS, Podaliri M. New Osseodensification Implant Site Preparation Method to Increase Bone Density in Low-Density Bone: In Vivo Evaluation in Sheep. Implant Dent 2016;25:24-31.

[7]. Albrektsson T, Jansson T (1986) Osseointegrated dental implants. Dent Clin North Am 30:151

[8]. Karthik K, Sivakumar, Sivaraj, Thangaswamy V. Evaluation of implant success: A review of past and present concepts. J Pharm Bioall Sci 2013;5:117-9. 